\title{
Custom total knee arthroplasty facilitates restoration of constitutional coronal alignment
}

\author{
Michel P. Bonnin ${ }^{1}$ - Lucas Beckers ${ }^{1}$ - Augustin Leon ${ }^{1}$. Jules Chauveau ${ }^{1}$ Jacobus H. Müller ${ }^{2}$ (D) Carsten O. Tibesku ${ }^{3}$. \\ Tarik Aït-Si-Selmi ${ }^{1}$
}

Received: 6 June 2020 / Accepted: 10 July 2020 / Published online: 17 July 2020

(c) The Author(s) 2020, corrected publication 2020

\begin{abstract}
Purpose To describe a strategy for coronal alignment using a computed tomography (CT) based custom total knee arthroplasty (TKA) system, and to evaluate the agreement between the planned and postoperative Hip-Knee-Ankle (HKA) angle, Femoral Mechanical Angle (FMA) and Tibial Mechanical Angle (TMA).

Methods From a consecutive series of 918 primary TKAs, 266 (29\%) knees received CT-based posterior-stabilized cemented custom TKA. In addition to a preoperative CT-scan, pre- and post-operative radiographs of weight-bearing long leg, anterior-posterior and lateral views of the knee were obtained, on which the FMA, TMA and HKA angles were measured. CTbased three-dimensional (3D) models enabled to correct for cases with bony wear by referring to the non-worn areas and to estimate the native pre-arthritic angles. The alignment technique aimed to preserve or restore constitutional alignment (CA) within predetermined limits, by defining a 'target zone' based on three criteria: 1 ) a $\pm 3^{\circ}$ (range $87^{\circ}-93^{\circ}$ ) primary tolerance for the femoral and tibial resections; 2 ) $\mathrm{a} \pm 2^{\circ}$ secondary tolerance for component obliquity, extending the bounds for FMA and TMA (range $85^{\circ}-95^{\circ}$ ); 3 ) a planned HKA angle range of $175^{\circ}-183^{\circ}$. Agreement between preoperative, planned and postoperative measurements of FMA, TMA and HKA angle were calculated using intra-class correlation coefficients (ICC). Results Preoperative radiograph and CT-scan measurements revealed that, respectively, 73 (28\%) and 103 (40\%) knees were in the 'target zone', whereas postoperative radiographs revealed that 217 (84\%) TKAs were in the 'target zone'. Deviation from the planned angles were $-0.5^{\circ} \pm 1.8^{\circ}$ for FMA, $-0.5^{\circ} \pm 1.8^{\circ}$ for TMA, and $-1.1^{\circ} \pm 2.1^{\circ}$ for HKA angle. Finally, the agreement between the planned and achieved targets, indicated by ICC, were good for FMA (0.701), fair for TMA (0.462) and fair for HKA angle (0.472).

Conclusion Using this strategy for coronal alignment, 84\% of custom TKAs were within the 'target zone' for FMA, TMA and HKA angles. These findings support the concepts of emerging personalized medicine technologies, and emphasise the importance of accurate strategies for preoperative planning, which are key to achieving satisfactory 'personalised alignment' that can further be improved by customisation of implant components.
\end{abstract}

Level of evidence IV.

Keywords Custom TKA · Total knee replacement · Total knee arthroplasty · HKA · FMA · TMA · Coronal alignment · Patient-specific

Electronic supplementary material The online version of this article (https://doi.org/10.1007/s00167-020-06153-8) contains supplementary material, which is available to authorized users.

\section{Jacobus H. Müller}

journals@ resurg.eu

1 Centre Orthopédique Santy, Hôpital Privé Jean Mermoz, Ramsay Santé, Lyon, France

2 ReSurg SA, Rue Saint Jean 22, 1260 Nyon, Switzerland

3 KniePraxis, Straubing, Germany

\section{Introduction}

Various knee alignment strategies for total knee arthroplasty (TKA) have been described and investigated, with the goals of improving functional outcomes and implant survival, but there remains little or no consensus about an optimal strategy. The different alignment philosophies can be considered as three main categories [39]: Systematic alignment (mechanical [21] or anatomical [20]), patient-specific alignment (kinematic 
[18]), and hybrid alignment (adjusted mechanical [41] or restricted kinematic [2]).

Mechanical alignment aims to restore a neutral tibiofemoral alignment, by making resections orthogonal to the tibial and femoral mechanical axes, regardless of a patient's prearthritic constitutional alignment (CA) [4]. Recently, it has been suggested that maintaining a residual varus alignment does not compromise TKA survival [1,34] and might improve functional outcomes [11, 27], whereas changing a preoperative varus to a postoperative valgus, or vice versa, could jeopardize functional outcomes [27]. By contrast, kinematic alignment aims to preserve or restore $\mathrm{CA}[4,26,29]$, by aligning implants to the native condylar and tibial joint lines, and by matching component thickness of the femoral condyles and tibia to that of resected bone [17, 19].

Although some studies have highlighted the limits of systematic neutral alignment $[1,11,24]$, the concept of patientspecific alignment continues to raise some controversial issues: First, with conventional radiographic preoperative planning, it is difficult to differentiate between constitutional and arthritic deformities [4, 6, 22, 28]. Second, the thresholds for acceptable postoperative residual varus or valgus remain unknown $[2,24,31,35]$. Third, component alignment and design are interrelated, and an individualized alignment associated with conventional implants can induce bone-implant mismatch or alter patellofemoral kinematics [31, 33, 37].

The theoretical benefits of custom TKA based on computed tomography (CT) reconstructions include the differentiation of constitutional versus arthritic bony deformities, as well as identification of the native femoral and tibial axes [11]. This concept has the potential to reduce boneimplant mismatch and preserve or restore CA within predetermined limits, to maintain the native overall phenotype while allowing correction of severe deformities [14-16]. The purpose of this study was to describe the strategy for coronal alignment using a CT-based custom TKA system and to evaluate the agreement between the planned and postoperative Hip-Knee-Ankle (HKA) angle, Femoral Mechanical Angle (FMA) and Tibial Mechanical Angle (TMA). The hypothesis was that custom TKA would enable achieving the intended coronal alignment in at least $80 \%$ of cases, irrespective of their preoperative deformity. The findings of this study would add to the evidence on emerging personalized medicine technologies, that can further be improved by customisation of implant components.

\section{Materials and methods}

\section{Patients}

From a consecutive series of 918 knees (905 patients) that received primary TKA between January and December

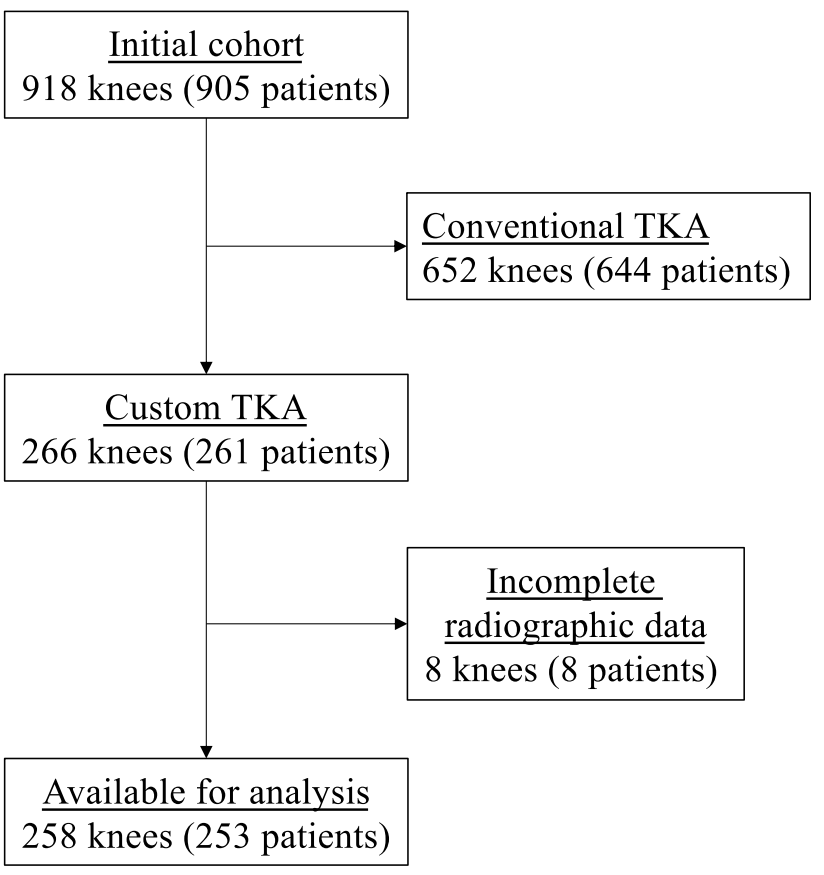

Fig. 1 Flowchart of the study cohort

2018 by three surgeons (MPB,TASS,COT) at two centres, 266 (29\%) knees (261 patients) received CT-based postero-stabilized cemented custom TKA (Origin ${ }^{\circledR}$ TKA, Symbios, Yverdon les bains, Switzerland). All patients had provided written informed consent for the use of their data and images for research and publishing purposes and the institutional review board approved the study in advance (IRB reference number: COS-RGDS-2019-10-004-BONNIN-M; Ramsay Santé Comité d'Ethique; + 33 (0)1 8786 22 97; Dr Sylviane Olschwang). Exclusion criteria for a custom TKA were: severe coronal deformities $>15^{\circ}$, stiff knees with extension deficit $>15^{\circ}$, flexion range $<90^{\circ}$, severe medial laxity $>10^{\circ}$, or severe lateral laxity $>15^{\circ}$. The final decision for using a custom TKA was based on the logistics of an 8 week waiting-period required for the design and manufacturing processes. The indications for surgery were medial osteoarthritis (OA) in 213 , lateral $\mathrm{OA}$ in 34, global OA in four, patellofemoral OA in eight and rheumatoid arthritis (RA) in seven knees. Eight knees were excluded due to unavailable postoperative long-leg radiographs, leaving a final cohort of 258 TKAs (118 men (five bilateral) and 135 women), with a mean age at index surgery of $70 \pm 9$ years (range 46-93 years) and a mean BMI of $29.5 \pm 13 \mathrm{~kg} / \mathrm{m}^{2}$ (range 18-44). Thirty knees had previous surgery: ligament reconstruction $(n=25)$, fixation of a distal femoral or proximal tibial fracture $(n=5)$, high tibial osteotomy (HTO, $n=6$ ), or tibial tubercle transfer $(n=3)$ (Fig. 1). 


\section{The custom TKA prosthesis}

All patients received a CT-scan (64-slice multidetector scanner, Siemens ${ }^{\circledR}$ Sensation, Munich, Germany) according to the manufacturer's protocol 8 weeks before surgery, on which the FMA, TMA and HKA angles were measured (Knee-Plan ${ }^{\circledR}$ software, Symbios, Yverdon les bains, Switzerland) to an accuracy of $1^{\circ}$ [15]. Three-dimensional (3D) models enabled to correct for cases with bony wear by referring to the non-worn areas and to estimate the native prearthritic angles [11]. The Origin ${ }^{\circledR}$ prosthesis is CE-marked and its design is based on a 3D analysis of bony anatomy, arthritic deformities and native limb alignment, as captured by the preoperative CT-scans. The femoral component reproduced the contours, sagittal radii of curvature and joint line obliquity of the native femur. The tibial baseplate reproduced the contours of the native tibial plateau, and the polyethylene insert had up to $2 \mathrm{~mm}$ difference in thickness between the medial and lateral compartments. Manufacturing of the femoral component was based on a conventional Cobalt-Chromium casting process, followed by machining and polishing, whereas the tibial baseplate was machined from Titanium alloy. Single-use patient-specific cutting guides were manufactured from polyamide using additive manufacturing technology.

\section{Alignment strategy}

Based on the classification of Hirschmann et al. [15, 16], which distinguishes 25 categories of HKA coronal alignment (five femoral phenotypes $\times$ five tibial phenotypes), the present study considered nine categories of HKA coronal alignment (three femoral phenotypes $\times$ three tibial phenotypes) (Fig. 2). This simplification was mainly achieved by disregarding the extent of varus or valgus deformity (minor if $<4.5^{\circ}$ or major if $>4.5^{\circ}$ ) within each bone, and by widening the range of what is considered to be neutral FMA $\left(91^{\circ}-95^{\circ}\right.$ instead of $\left.91.5^{\circ}-94.5^{\circ}\right)$, and what is considered to be neutral TMA $\left(85^{\circ}-89^{\circ}\right.$ instead of $\left.85.5^{\circ}-88.5^{\circ}\right)$.

The alignment technique for the Origin ${ }^{\circledR}$ TKA aims to preserve or restore $\mathrm{CA}$ within predetermined limits, allow correction of severe deformities, and maintain the native overall phenotype, by defining a 'target zone' based on three criteria (Fig. 3):
Fig. 2 This matrix represents FMA on the horizontal axis and TMA on the vertical axis. Varus femurs were defined as FMA $<91^{\circ}$ and Valgus femurs as FMA $>95^{\circ}$; Varus tibias were defined as TMA $<85^{\circ}$ and Valgus tibias as TMA $>89^{\circ}$. Overall limb alignment was considered in varus if HKA angle $<177^{\circ}$ and in valgus if HKA angle $>183^{\circ}$. Finally nine morphotypes were defined based on FMA and TMA

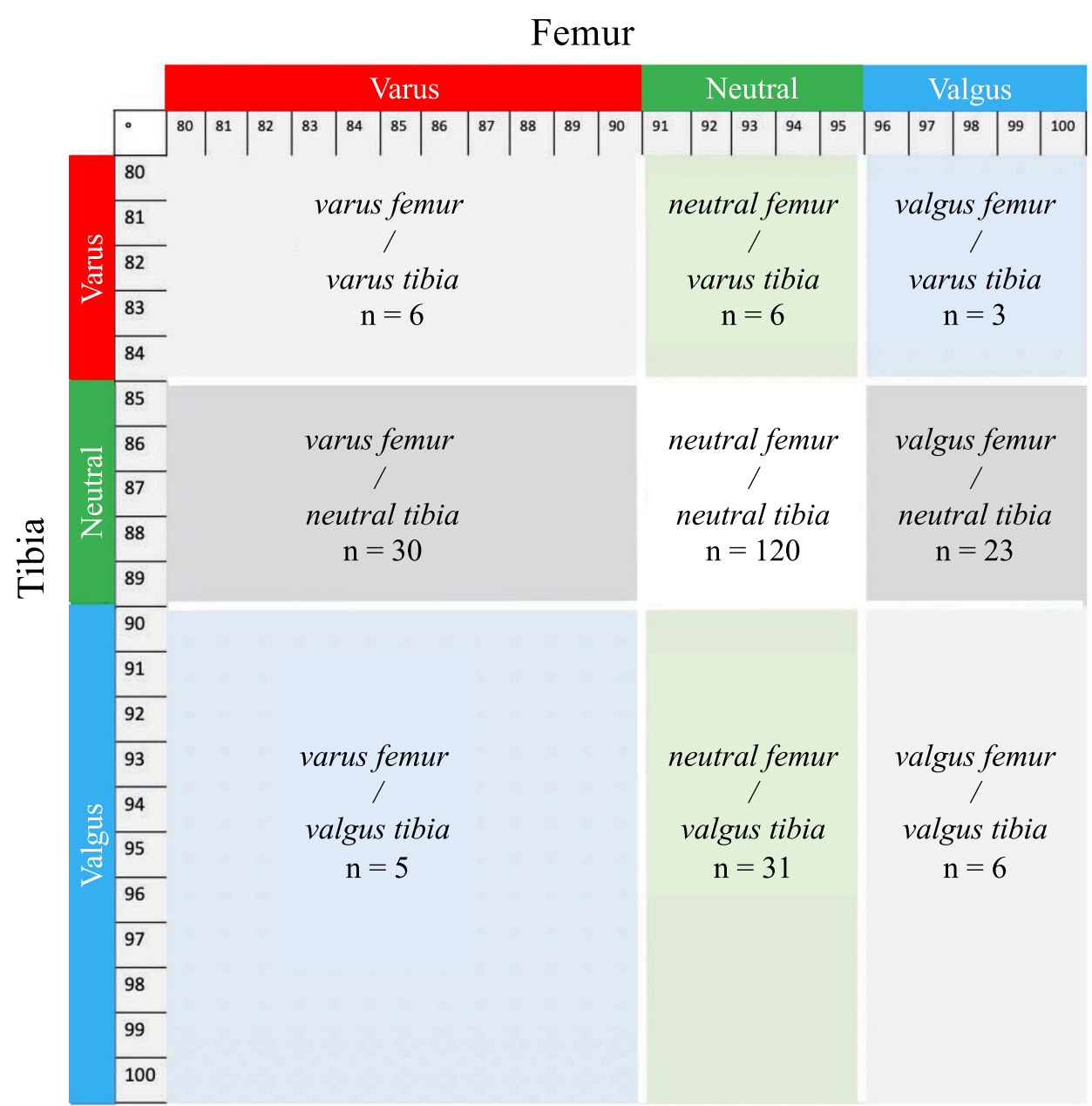


1. A primary tolerance of $\pm 3^{\circ}$ for the femoral and tibial cuts, which were planned to achieve a range of mechanical angles from $87^{\circ}-93^{\circ}$, depending on the pre-arthritic phenotype [12, 35, 36].

2. A secondary tolerance of $\pm 2^{\circ}$ for implant obliquity (polyethylene insert and femoral condyles), which extended the total range of mechanical angles from $85^{\circ}-95^{\circ}$, to remain as close as possible to the pre-arthritic phenotype [32].

3. A planned HKA angle within the range of $175^{\circ}-183^{\circ}$ [27].

The application of the three criteria resulted in a 'target zone' (Fig. 4). When the preoperative FMA and TMA were inside the 'target zone', the planned alignment should correspond to CA, while when the preoperative FMA and TMA were outside of the 'target zone', the planned alignment was corrected to the closest configuration within the 'target zone', to maintain the native overall phenotype (Fig. 5).

\section{Surgical technique}

All patients were operated using a medial parapatellar approach. Femoral and tibial resections were made using the custom cutting guides by a 'femur-first' technique. Soft tissue balance was then evaluated with a dynamic spacer, and if necessary, the level of tibial resection was adjusted

$\mathbf{a}$

Femur

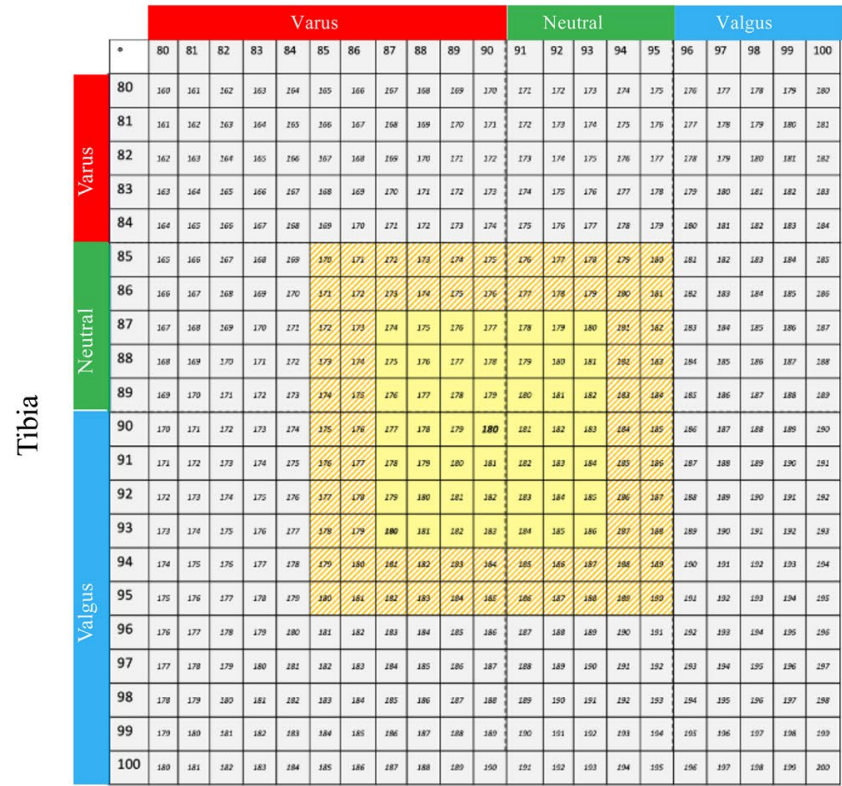

Fig. 3 a Matrix shows the alignment possibilities offered by the Origin ${ }^{\circledR}$ system. A primary tolerance of $\pm 3^{\circ}$ for the femoral and tibial cuts, which were planned to achieve a range of mechanical angles from $87^{\circ}$ to $93^{\circ}$ (yellow area). A secondary tolerance of $\pm 2^{\circ}$ by making a 'recut' using a dedicated guide. Once all bone surfaces were prepared, the tibia and then the femur and patella were cemented. Immediate full weight-bearing was authorised, and rehabilitation began on the same day of surgery.

\section{Radiographic analysis}

Pre- and postoperative radiographs of weight-bearing long leg, anterior-posterior and lateral views of the knee and a skyline view of the patella were obtained for each patient. Radiographic follow-up occurred at 4 months. Pre- and postoperative alignment was measured on the weight-bearing long-leg radiographs: The mechanical Femoral Axis (mFA) was defined as the line joining the centre of the femoral head and the centre of the intercondylar notch. The FMA was measured medially between the mFA and the distal condylar line. The mechanical Tibial Axis (mTA) was defined as the line joining the middle of the tibial spines and the centre of the tibiotalar joint. The TMA was then measured medially between the tibial joint line and the mTA. Finally, the HKA angle was measured medially between mFA and mTA. Radiographic measurements were performed (MPB) on an online picture archiving and communication system (GXD5 PACS, groupe NGI, Lyon, France) with a measurement accuracy of $0.1^{\circ}$, and rounded to the nearest whole number.

b

Femur

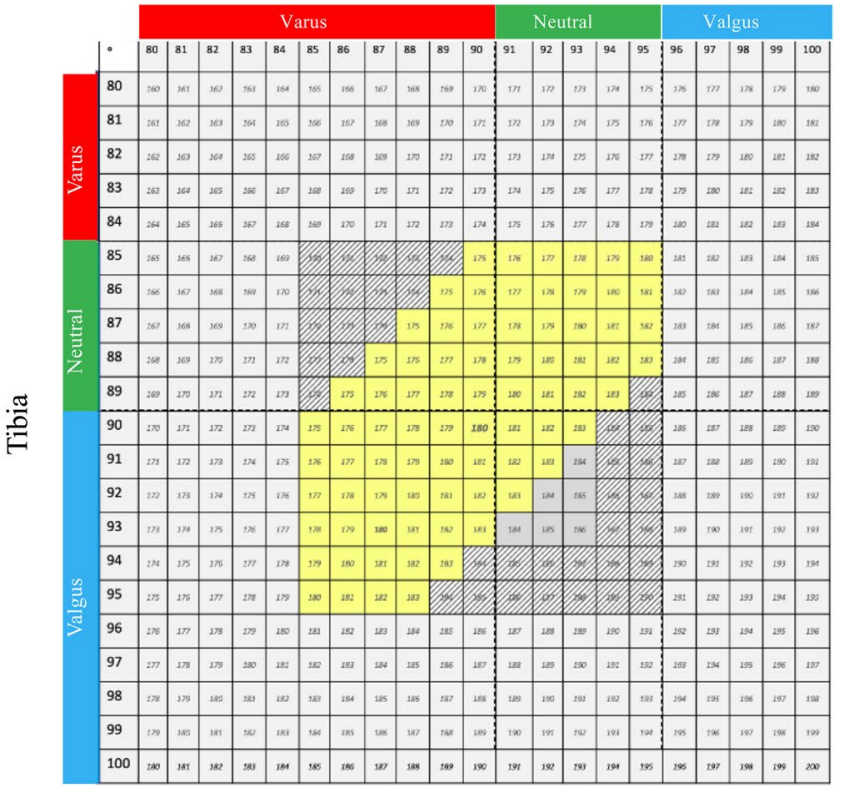

for implant obliquity (polyethylene insert and femoral condyles), which extended the total range of mechanical angles from $85^{\circ}$ to $95^{\circ}$ (Orange area). b 'Target zone' (Yellow area) respecting the $85^{\circ}$ to $95^{\circ}$ range for FMA and TMA, and the $175^{\circ}$ to $183^{\circ}$ range for HKA angle 
Fig. 4 When the preoperative FMA and TMA are within the 'target zone', the constitutional alignment is maintained postoperatively. When the preoperative FMA and TMA are outside the 'target zone', the alignment is corrected

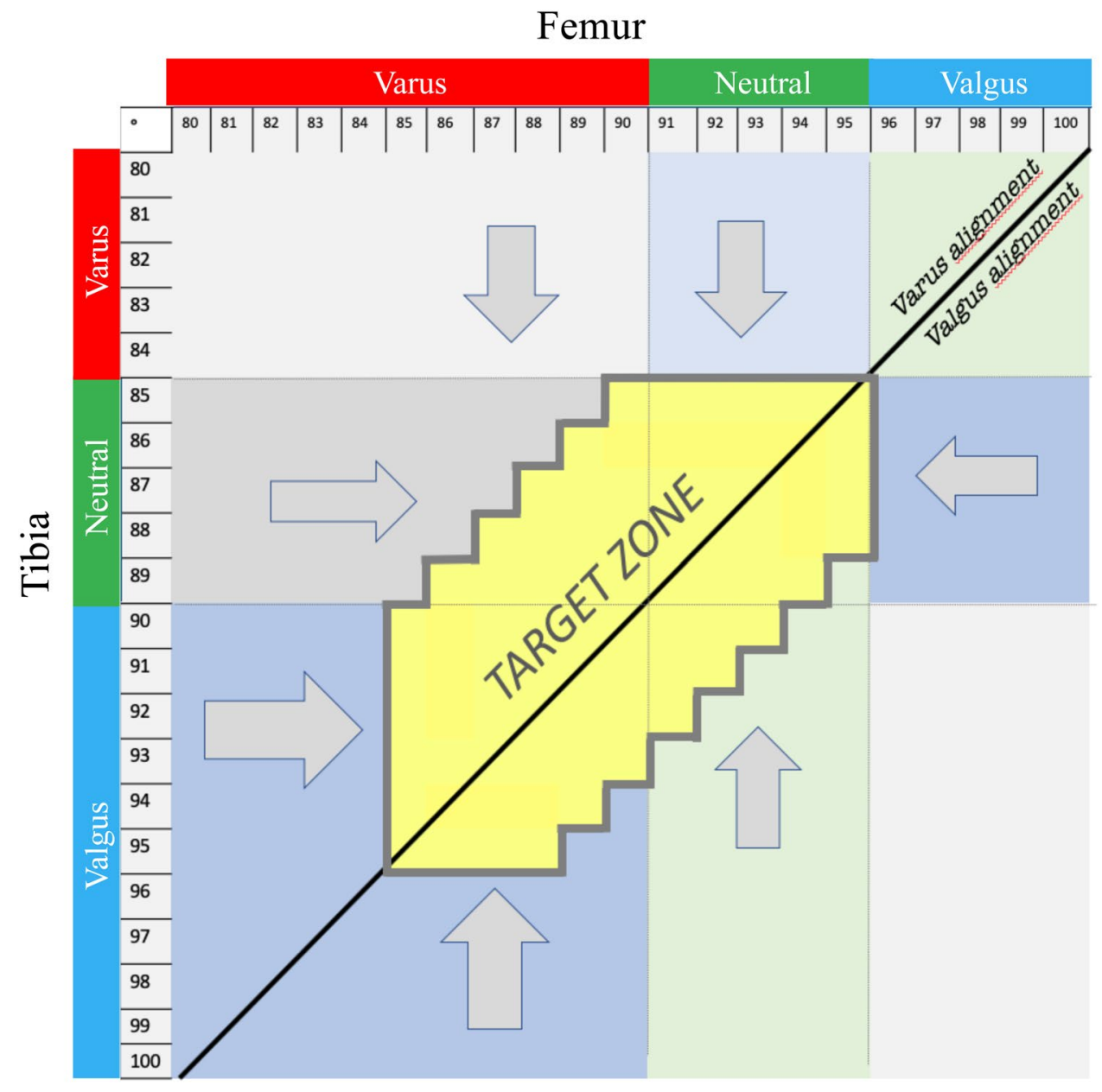

\section{Statistical analysis}

Shapiro-Wilk tests were used to assess the normality of data distributions. For non-Gaussian quantitative data, differences between groups were evaluated using Wilcoxon rank sum tests (Mann-Whitney $U$ test) and Kruskal-Wallis tests. For categorical data, differences between groups were evaluated using Chi-squared tests. Agreement between preoperative, planned and postoperative measurements of FMA, TMA and HKA angle were calculated using intra-class correlation coefficients (ICC), which can be interpreted as follows: $<0.40$ poor; $0.40-0.59$ fair; 0.60-0.74 good; 0.75-1.00 excellent. A second observer (LB) repeated radiographic measurements of pre- and post-operative FMA, TMA and HKA angles for $25(10 \%)$ knees. The interobserver agreement calculated using ICC was excellent (ICC 0.774-0.994) for all measurements. Statistical analyses were performed using $\mathrm{R}$ version 3.3.2 (R Foundation for Statistical Computing, Vienna, Austria). $p$ values $<0.05$ were considered statistically significant.

\section{Results}

Preoperative coronal alignment as measured on CT-scans and radiographs were, respectively, $92.7^{\circ} \pm 2.5^{\circ}$ and $92.2^{\circ} \pm 2.6^{\circ}$ (n.s.) for FMA, 87. $0^{\circ} \pm 2.7^{\circ}$ and $86.5^{\circ} \pm 3.0^{\circ}$ $(p=0.038)$ for TMA, and $176.9^{\circ} \pm 5.0^{\circ}$ and $175.6^{\circ} \pm 6.2^{\circ}$ $(p<0.001)$ for HKA angle (Table 1). The most common phenotype, as measured from the preoperative CT-scans, was 'neutral femur and neutral tibia' $(n=120,47 \%)$, and the least common was 'valgus femur and varus tibia' $(n=3$, 1\%) (Fig. 2). The proportion of knees that were in the 'target zone' according to preoperative radiograph and CT-scan measurements were, respectively, 73 (28\%) and 103 (40\%).

Deviation between the planned and postoperative angles were $-0.5^{\circ} \pm 1.8^{\circ}$ for FMA, $-0.5^{\circ} \pm 1.8^{\circ}$ for TMA, and $-1.1^{\circ} \pm 2.1^{\circ}$ for HKA angle (Table 2). A total of 217 (84\%) TKAs were in the 'target zone', based on the postoperative radiograph measurements (Fig. 6). Finally, the agreement between the planned and achieved targets, indicated by ICC, were good for FMA, but fair for TMA and HKA angle. 
a

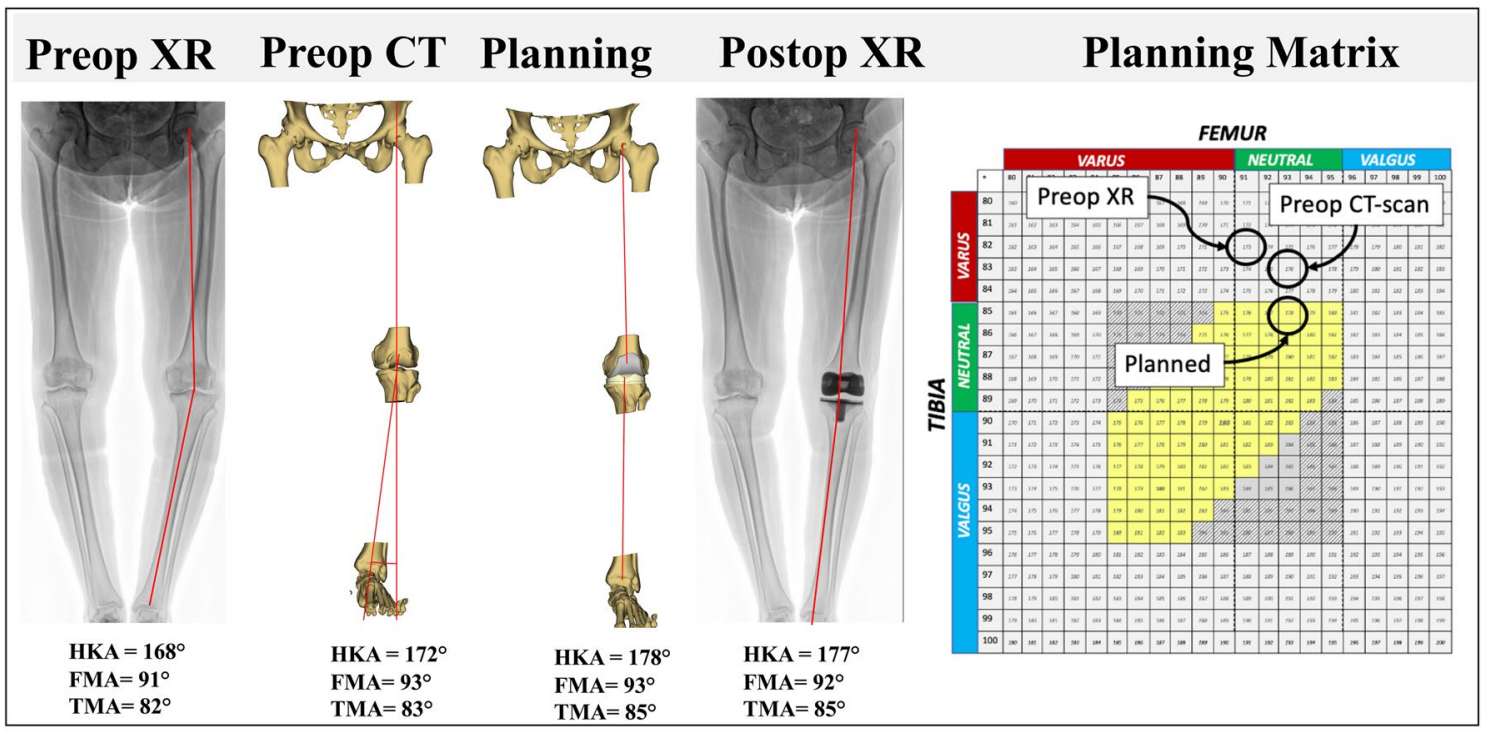

b

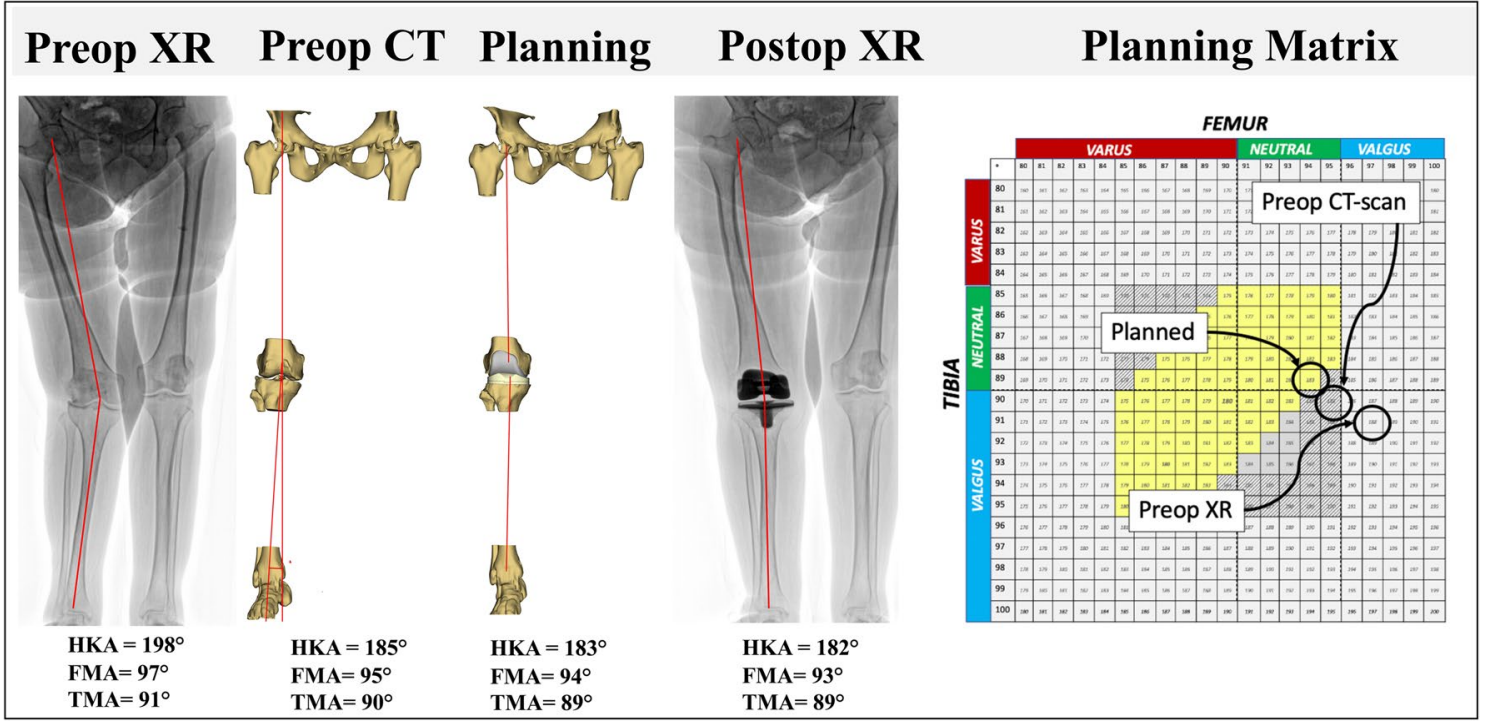

Fig. 5 a In this patient, the global radiograph varus deformity is a combination of arthritic deformity (bone wear and laxity) and constitutional deformity $\left(\mathrm{HKA}\right.$ angle $\left.=168^{\circ}\right)$. Constitutional alignment is outside the 'target zone' $\left(\mathrm{FMA}=91^{\circ}\right.$ and $\left.\mathrm{TMA}=82^{\circ}\right)$. The planning corrects the deformity to the 'target zone' $\left(\mathrm{FMA}=92^{\circ}, \mathrm{TMA}=85^{\circ}\right.$, HKA angle $=177^{\circ}$ ). $\mathbf{b}$ In this patient, the global radiograph valgus

The tibial resection level was adjusted intraoperatively by a 'recut' in 118 knees (46\%) to achieve appropriate soft tissue balance (Table 2). The proportion of TKAs that were in the 'target zone' for cases that required a 'recut' and those that did not was, respectively, 97 (82\%) and 120 (86\%). Finally, the agreement between the planned and achieved targets in knees that required recuts, indicated by ICC, remained good for FMA, fair for TMA, but changed to poor for HKA angle. deformity is a combination of arthritic deformity (bone wear and laxity) and constitutional deformity (HKA angle $=198^{\circ}$ ). Constitutional alignment is outside the 'target zone' $\left(\mathrm{FMA}=97^{\circ}\right.$ and $\left.\mathrm{TMA}=91^{\circ}\right)$. The planning corrects the deformity to the 'target zone' (FMA $=93^{\circ}$, $\mathrm{TMA}=89^{\circ}, \mathrm{HKA}$ angle $=182^{\circ}$ )

\section{Discussion}

The most important finding of the present study was that, using this strategy for coronal alignment, $84 \%$ of custom TKAs were within the 'target zone' in terms of FMA, TMA and HKA angles. Beyond the reliability of preoperative planning tools as well as intraoperative patient-specific instruments and techniques, the present study encompasses the main pillars for success of TKA, including personalised 
Table 1 Preoperative coronal alignment according to radiograph and CT measurements

\begin{tabular}{llll}
\hline & $\begin{array}{l}\text { Mean } \pm \text { SD } \\
\text { ICC }\end{array}$ & $\begin{array}{l}\text { (Range) } \\
(95 \% \text { CI })\end{array}$ & $\begin{array}{l}p \text { values } \\
\text { (Radio- } \\
\text { graph vs. } \\
\text { CT) }\end{array}$ \\
\hline FMA & & & \\
Radiograph (deg) & $92.2 \pm 2.6$ & $(84-99)$ & n.s \\
CT (deg) & $92.7 \pm 2.5$ & $(87-99)$ & \\
Delta (deg) & $0.4 \pm 1.5$ & $(-4-5)$ & \\
Agreement & 0.827 & $(0.767-0.870)$ & \\
TMA & & & \\
Radiograph (deg) & $86.5 \pm 3.0$ & $(79-95)$ & \\
CT (deg) & $87.0 \pm 2.7$ & $(80-94)$ & \\
Delta (deg) & $0.5 \pm 1.6$ & $(-4-7)$ & \\
Agreement & 0.830 & $(0.764-0.876)$ & \\
HKA angle & & & $<0.001$ \\
Radiograph (deg) & $175.6 \pm 6.2$ & $(162-195)$ & \\
CT (deg) & $176.9 \pm 5.0$ & $(165-192)$ & \\
Delta (deg) & $1.3 \pm 2.8$ & $(-11-13)$ & \\
Agreement & 0.859 & $(0.762-0.910)$ & \\
\hline
\end{tabular}

FMA femoral mechanical angle; TMA tibial mechanical angle, $H K A$ hip knee ankle; $C T$ computed tomography; $S D$ standard deviation; ICC intra-class correlation cofficient; $C I$ confidence interval; deg degree; n.s not signifcant

limb alignment, and implant geometry to reproduce bony contours and radii of curvature. The present findings support the concepts of emerging personalized medicine technologies [13, 42], and their clinical relevance emphasises the importance of detailed and accurate strategies for preoperative planning, which are key to achieving satisfactory 'personalised alignment' that can further be improved by customisation of implant components.

Several other findings emerge from this study. First, it outlines the limits of radiographic analysis for the understanding of patient phenotypes. The conventional 2D planning can only analyse the global limb deformity but can hardly differentiate its arthritic part (bone wear and laxity) from its constitutional part, the latter remaining unknown $[6,22,28]$. Additionally, any rotation of the leg can alter the long-leg radiograph accuracy [28]. The CT-scan allows to analyse the femur and tibia separately in their true frontal planes, and to deduce the native pre-arthritic architecture of the limb despite the bone wear. Second, this study describes a new (re)alignment strategy, based on an accurate planning, using the new possibilities offered by customization. The matrix approach relies on a rationalized 'individual alignment' rather than a 'systematic alignment' strategy and on the definition of a 'target zone' rather than a 'target value'. The thresholds defining the 'target zone' intend to remain within safe limits in terms of tribology and fixation for the bone cuts inclination [12], the coronal asymmetry of the implants [3,23], the joint line obliquity [32] and the realignment in valgus knees. These thresholds may be modified depending on findings in future studies. Third, it demonstrates the technical feasibility of a new process, providing a pre-manufacturing roadmap for engineers, a traceable planning for surgeons and finally individualized implants adapted to the bony anatomy and alignment. Previous series of custom TKA were based on a 'systematic alignment' concept intending to realign all patients to $180^{\circ}$, and this is the first process that aims to restore both the native shape of the bones and the constitutional alignment (CA) [3, 23].

Historically, MA has been considered as the gold standard, with several studies demonstrating its superiority for TKA survival $[12,36]$. Surgeons learned how to tackle ligament imbalance due to orthogonal cuts with technical tricks such as ligament releases or external rotation of the femoral component [5], all of which are 'palliative solutions', compensating for the modification of the CA. Recently, several factors challenged the dogma of MA. Biomechanical studies have reported a discrepancy between the radiographic static axis of the lower limb and the functional dynamic axis during gait [40]. Large population studies demonstrated that native limb alignment is highly variable $[4,14,30]$. The rate of dissatisfaction due to residual pain or unmet expectations after TKA is reported within the range of 5-25\%, [7, 8 ] and could be attributed to the lack of restoration of the native anatomy $[4,16,30]$. Long term studies reported that patients outside the $180^{\circ} \pm 3^{\circ}$ range had similar survivorship as neutrally aligned patients $[1,11,34]$, and that a residual varus may improve function [11,27], whereas a change from varus to valgus (or vice versa) could reduce patient satisfaction [27].

Progressively, the need of a kind of personalization appeared $[4,29]$ and several surgeons unconsciously modified their practice from MA towards partial preservation of the preoperative deformity [26]. The epitome of this evolution is kinematic alignment (KA), where the native alignment is precisely reproduced, with a strict matching between resected bones and implant thicknesses [17]. Several authors compared MA and KA [9, 11, 44], but it is still unclear whether the differences are clinically relevant [25] and the place of KA remain controversial with three main limitations [44]. First, alignment and implant design are interrelated, and the variations of implant positioning in KA may induce bone-implant mismatch [37], trochlear malalignment [38] or femoral malrotation [33]. Second, the original KA technique is based purely on intraoperative measurements rather than on precise preoperative planning, and may be subject to surgeon experience [17]. Robotic assistance may in the future fill the gap between preoperative planning and surgical execution $[9,10]$. Third, the limits of acceptable residual coronal 


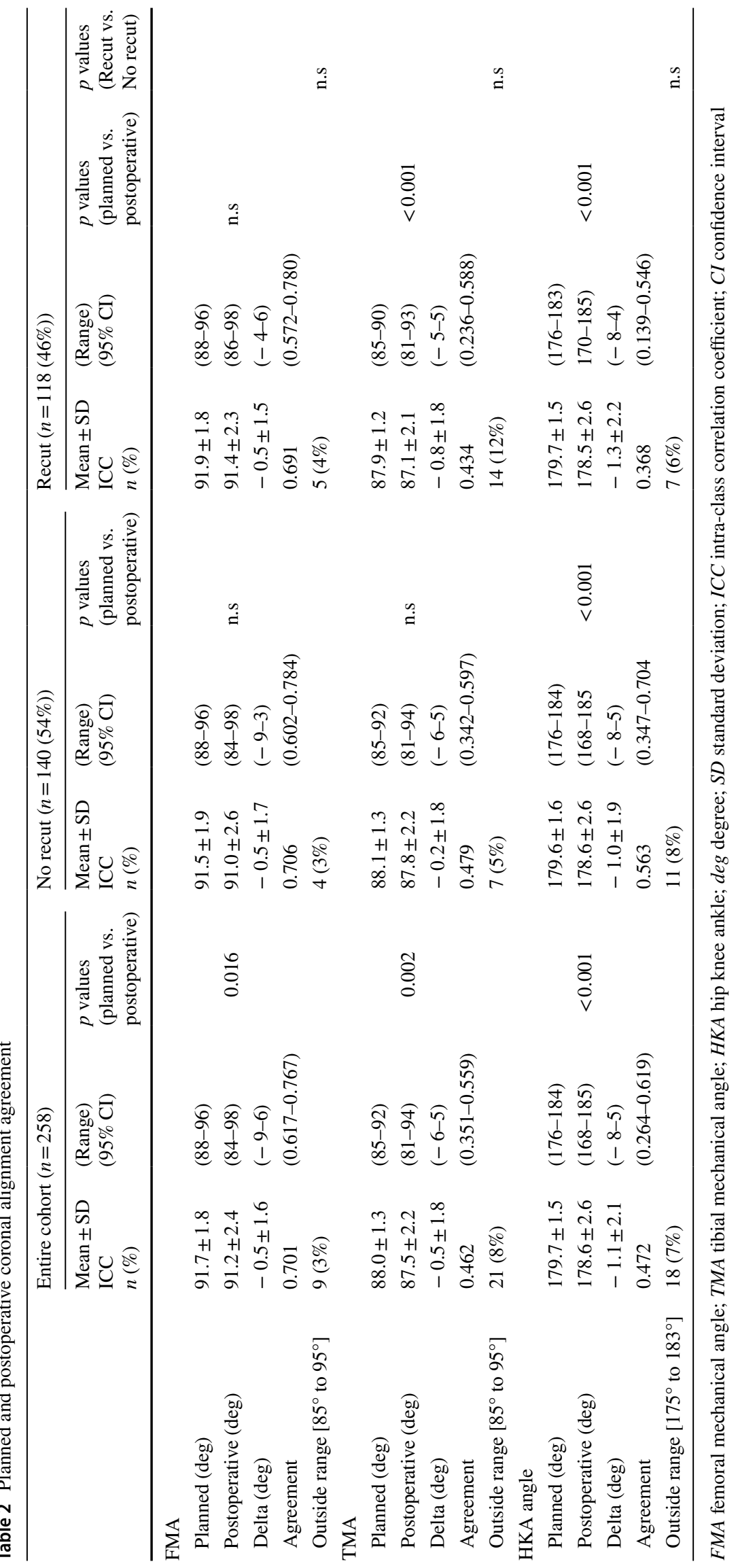



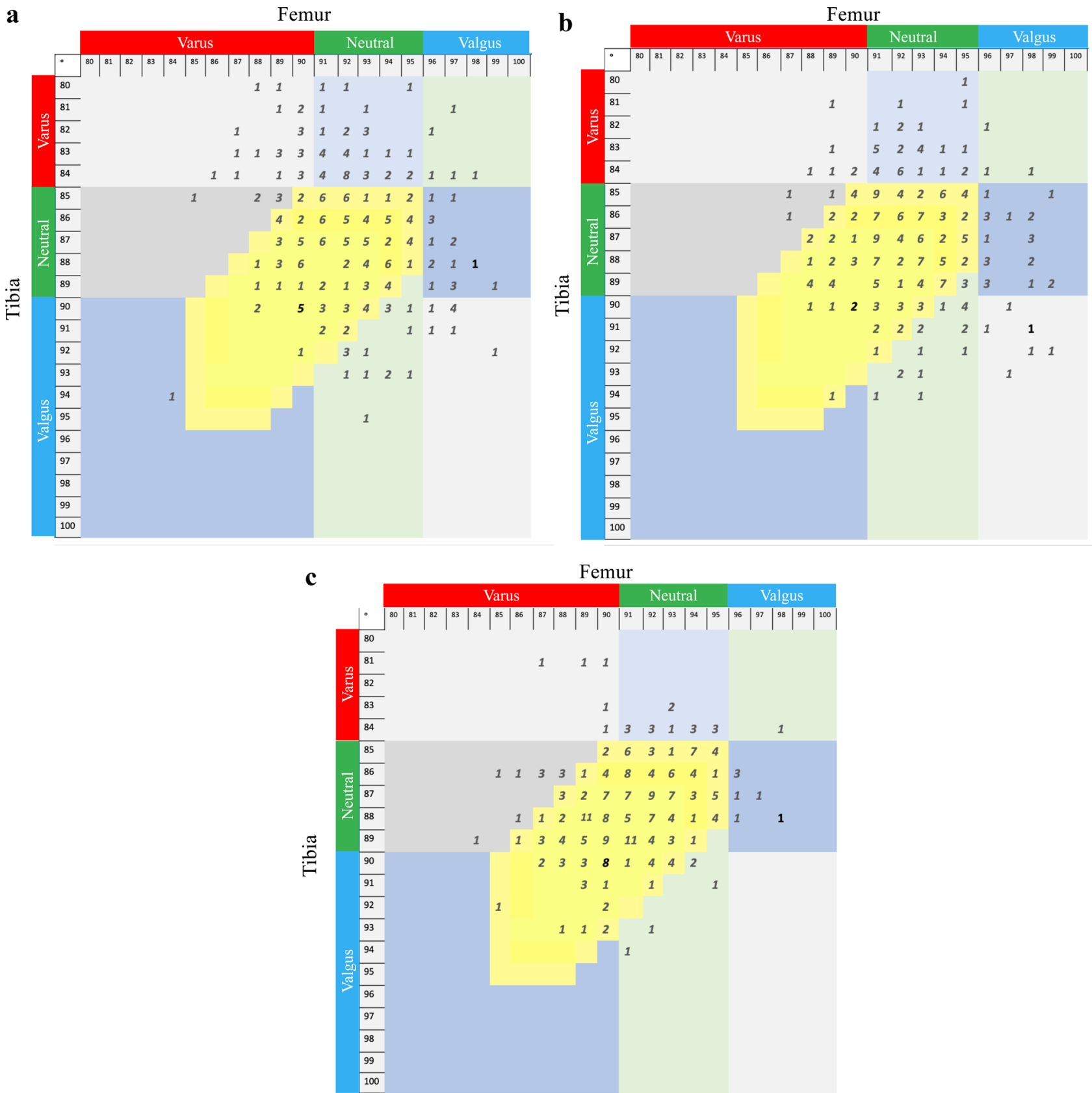

Fig. 6258 knees of the series are included in these matrices with FMA and TMA measured on: a preoperative long-leg radiographs, b preoperative CT-scans, and $\mathbf{c}$ postoperative long-leg radiographs

deformity in TKA are still not clearly understood. Parratte et al. [34] and Abdel et al. [1] observed that outliers and neutrally aligned patients had similar long term survivorship, and Howell et al. [19] reported 98.4\% survivorship (aseptic failures) at 10 year follow-up with KA. However, many authors report higher rates of failure in patients with residual varus $[12,27,36]$. It is worth noting that while some authors investigated the influence of residual postoperative deformities with respect to the preoperative radiographic deformity $[26,35]$, few investigated the role of constitutional versus arthritic deformity [43] (Fig. 7). It is also unclear whether a residual deviation has the same consequences if observed at the femur or at the tibia [27]. Based on all these unanswered questions, Almaawi et al. [2] described restricted Kinematic Alignment (rKA) which maintains a $180^{\circ} \pm 3^{\circ}$ range for HKA with a $90^{\circ} \pm 5^{\circ}$ range for FMA and TMA. The concept of custom TKA has also been explored with iTotal ${ }^{\circledR}$ TKA (ConforMIS, Billerica, MA, USA), but using a concept of Systematic Alignment, the target being a $180^{\circ}$ global alignment $[3,23]$. 
$\mathbf{a}$

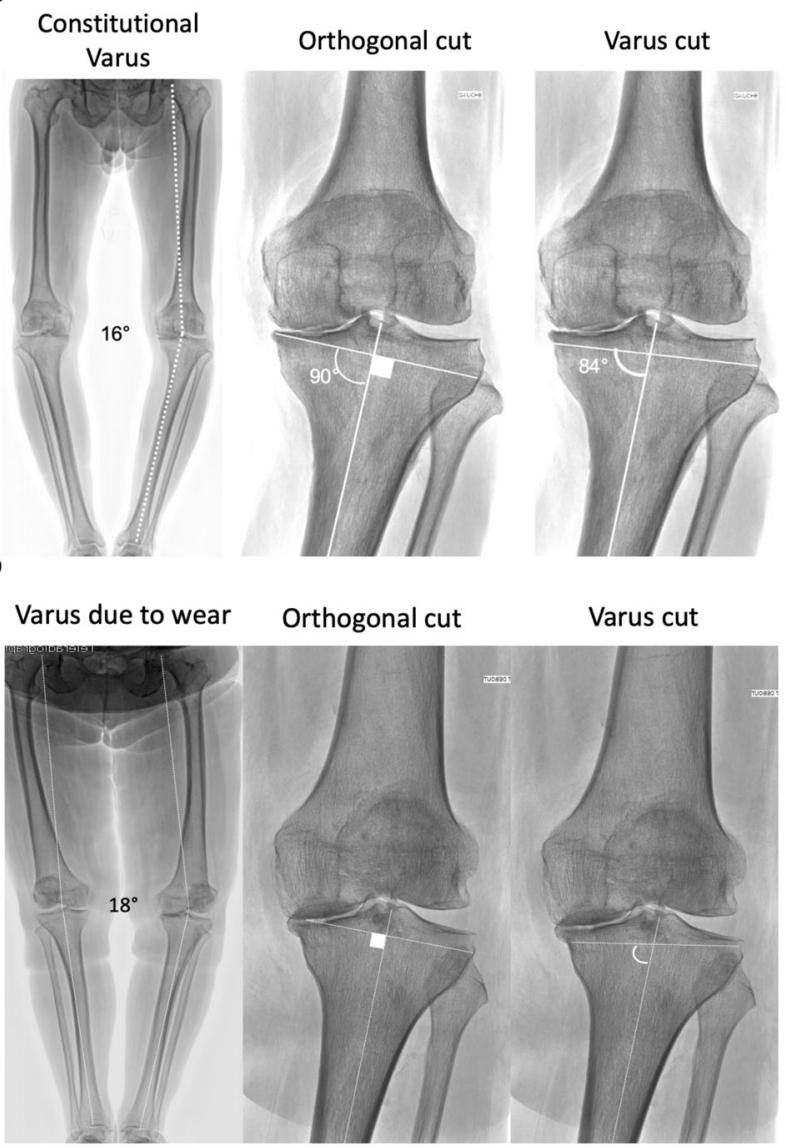

Fig. 7 Examples where varus cuts have different consequences. In patient A, bone wear is minimal and most of the global varus deformity $\left(16^{\circ}\right)$ is due to its native architecture (constitutional varus). An orthogonal cut induce an asymmetric resection, in a weak zone of the lateral tibial plateau. With a varus cut there is less asymmetry and the bone cut remain in the subchondral area. In patient $\mathrm{B}$, bone wear is severe and most of the global varus deformity $\left(18^{\circ}\right)$ is due to the bone loss (no constitutional varus). An orthogonal cut corrects the bone loss and do not induce asymmetric resection. A varus cut is very oblique with respect to the tibial shape and is situated below the subchondral area on the medial tibial plateau

The results of the present study need to be interpreted in light of the following limitations. First, the discrepancy between planned and postoperative alignment is partly attributable to the learning curve as this is a consecutive continuous series including all patients, from three senior surgeons who were trained with traditional instrumentation. Second, a tibial bone recut was performed in $46 \%$ of the procedures and a lack of accuracy of the re-cutting guides can explain discrepancies between planned and postoperative angles, particularly in patients with weak bones. Third, in this retrospective case series, there was a selection bias, since the indications for custom TKA were limited to patients without severe deformities with good preoperative ROM. Fourth, the absolute number of patients that were excluded based on the exclusion criteria, and the absolute number of patients that were excluded due to the waiting period are not reported. Finally, long term survivorship, clinical and functional outcomes are still to be confirmed; therefore, this study cannot conclude on the superiority or inferiority of custom TKA. The results do indicate the feasibility of using a more personalized strategy for coronal alignment in TKA.

\section{Conclusion}

Using the present strategy for coronal alignment, $84 \%$ of custom TKAs were within the 'target zone' for FMA, TMA and HKA angles. These encouraging findings support the concepts of emerging personalized medicine technologies, and their clinical relevance emphasises the importance of detailed and accurate strategies for preoperative planning, which are key to achieving satisfactory 'personalised alignment' that can further be improved by customisation of implant components.

Author contributions MPB: study design, literature review, data collection, manuscript writing, figures. LB: data collection, manuscript editing. AL: data collection, manuscript editing. JC: data collection, manuscript editing. JHM: statistical analysis, manuscript writing, figures and tables. COT: study design, data collection, manuscript editing. TASS: study design, data collection, manuscript editing.

Funding The authors are grateful to Ramsay Santé for funding the statistical analysis and manuscript preparation for this study. The authors are grateful to Mo Saffarini for assistance with study design and interpretation of findings.

\section{Compliance with ethical standards}

Conflict of interest MPB reports personal fees from DePuy Synthes, Wright Medical, Integra and Symbios. LB has nothing to declare. AL has nothing to declare. JC has nothing to declare. JHM has nothing to declare. COT reports personal fees from Symbios and Smith \& Nephew. TASS reports personal fees from DePuy-Synthes and from Symbios.

Ethical approval All patients had provided written informed consent for the use of their data and images for research and publishing purposes and the institutional review board approved the study in advance (IRB reference number: COS-RGDS-2019-10-004-BONNIN-M; Ramsay Santé Comité d'Ethique; +33 (0)1 878622 97; Dr Sylviane Olschwang).

Open Access This article is licensed under a Creative Commons Attribution 4.0 International License, which permits use, sharing, adaptation, distribution and reproduction in any medium or format, as long as you give appropriate credit to the original author(s) and the source, provide a link to the Creative Commons licence, and indicate if changes were made. The images or other third party material in this article are included in the article's Creative Commons licence, unless indicated 
otherwise in a credit line to the material. If material is not included in the article's Creative Commons licence and your intended use is not permitted by statutory regulation or exceeds the permitted use, you will need to obtain permission directly from the copyright holder. To view a copy of this licence, visit http://creativecommons.org/licenses/by/4.0/.

\section{References}

1. Abdel MP, Ollivier M, Parratte S, Trousdale RT, Berry DJ, Pagnano MW (2018) Effect of postoperative mechanical axis alignment on survival and functional outcomes of modern total knee arthroplasties with cement: a concise follow-up at 20 years. J Bone Joint Surg Am 100(6):472-478

2. Almaawi AM, Hutt JRB, Masse V, Lavigne M, Vendittoli PA (2017) The impact of mechanical and restricted kinematic alignment on knee anatomy in total knee arthroplasty. J Arthroplasty 32(7):2133-2140

3. Arbab D, Reimann P, Brucker M, Bouillon B, Luring C (2018) Alignment in total knee arthroplasty-A comparison of patient-specific implants with the conventional technique. Knee 25(5):882-887

4. Bellemans J, Colyn W, Vandenneucker H, Victor J (2012) The Chitranjan Ranawat award: is neutral mechanical alignment normal for all patients? The concept of constitutional varus. Clin Orthop Relat Res 470(1):45-53

5. Berger RA, Crossett LS, Jacobs JJ, Rubash HE (1998) Malrotation causing patellofemoral complications after total knee arthroplasty. Clin Orthop Relat Res 356:144-153

6. Bonnin M, Chambat P (2004) Current status of valgus angle, tibial head closing wedge osteotomy in media gonarthrosis. Orthopade 33(2):135-142

7. Bonnin M, Laurent JR, Parratte S, Zadegan F, Badet R, Bissery A (2010) Can patients really do sport after TKA? Knee Surg Sports Traumatol Arthrosc 18(7):853-862

8. Bourne RB, Chesworth BM, Davis AM, Mahomed NN, Charron KD (2010) Patient satisfaction after total knee arthroplasty: who is satisfied and who is not? Clin Orthop Relat Res 468(1):57-63

9. Calliess T, Bauer K, Stukenborg-Colsman C, Windhagen H, Budde S, Ettinger M (2017) PSI kinematic versus non-PSI mechanical alignment in total knee arthroplasty: a prospective, randomized study. Knee Surg Sports Traumatol Arthrosc 25(6): 1743-1748

10. Calliess T, Ettinger M, Savov P, Karkosch R, Windhagen $H$ (2018) Individualized alignment in total knee arthroplasty using image-based robotic assistance: video article. Orthopade 47(10):871-879

11. Dossett HG, Estrada NA, Swartz GJ, LeFevre GW, Kwasman BG (2014) A randomised controlled trial of kinematically and mechanically aligned total knee replacements: two-year clinical results. Bone Joint J 96-b(7):907-913

12. Fang DM, Ritter MA, Davis KE (2009) Coronal alignment in total knee arthroplasty: just how important is it? J Arthroplasty 24(6 Suppl):39-43

13. Hirschmann MT, Friederich NF, Becker R, Karlsson J (2019) Personalised medicine in knee arthroplasty: we need more science! Knee Surg Sports Traumatol Arthrosc 27(5):1357-1358

14. Hirschmann MT, Hess S, Behrend H, Amsler F, Leclercq V, Moser LB (2019) Phenotyping of hip-knee-ankle angle in young non-osteoarthritic knees provides better understanding of native alignment variability. Knee Surg Sports Traumatol Arthrosc 27(5):1378-1384

15. Hirschmann MT, Moser LB, Amsler F, Behrend $\mathrm{H}$, Leclercq V, Hess S (2019) Phenotyping the knee in young non-osteoarthritic knees shows a wide distribution of femoral and tibial coronal alignment. Knee Surg Sports Traumatol Arthrosc 27(5):1385-1393

16. Hirschmann MT, Moser LB, Amsler F, Behrend H, Leclerq V, Hess S (2019) Functional knee phenotypes: a novel classification for phenotyping the coronal lower limb alignment based on the native alignment in young non-osteoarthritic patients. Knee Surg Sports Traumatol Arthrosc 27(5):1394-1402

17. Howell SM (2019) Calipered kinematically aligned total knee arthroplasty: an accurate technique that improves patient outcomes and implant survival. Orthopedics 42(3):126-135

18. Howell SM, Hull ML (2012) Kinematic alignment in total knee arthroplasty. Insall and Scott Surgery of the Knee Philadelphia. Elsevier, PA, pp 1255-1268

19. Howell SM, Shelton TJ, Hull ML (2018) Implant survival and function ten years after kinematically aligned total knee arthroplasty. J Arthroplasty 33(12):3678-3684

20. Hungerford DS, Kenna RV, Krackow KA (1982) The porouscoated anatomic total knee. Orthop Clin North Am 13(1):103-122

21. Insall JN, Binazzi R, Soudry M, Mestriner LA (1985) Total knee arthroplasty. Clin Orthop Relat Res NO 192:13-22

22. Jenny JY, Boeri C, Ballonzoli L, Meyer N (2005) Difficulties and reproducibility of radiological measurement of the proximal tibial axis according to Levigne. Rev Chir Orthop Reparatrice Appar Mot 91(7):658-663

23. Levengood GA, Dupee J (2018) Accuracy of coronal plane mechanical alignment in a customized, individually made total knee replacement with patient-specific instrumentation. J Knee Surg 31(8):792-796

24. Liu HX, Shang P, Ying XZ, Zhang Y (2016) Shorter survival rate in varus-aligned knees after total knee arthroplasty. Knee Surg Sports Traumatol Arthrosc 24(8):2663-2671

25. Luo Z, Zhou K, Peng L, Shang Q, Pei F, Zhou Z (2019) Similar results with kinematic and mechanical alignment applied in total knee arthroplasty. Knee Surg Sports Traumatol Arthrosc. https:// doi.org/10.1007/s00167-019-05584-2

26. Luyckx T, Vanhoorebeeck F, Bellemans J (2015) Should we aim at undercorrection when doing a total knee arthroplasty? Knee Surg Sports Traumatol Arthrosc 23(6):1706-1712

27. Magnussen RA, Weppe F, Demey G, Servien E, Lustig S (2011) Residual varus alignment does not compromise results of TKAs in patients with preoperative varus. Clin Orthop Relat Res 469(12):3443-3450

28. McGrory JE, Trousdale RT, Pagnano MW, Nigbur M (2002) Preoperative hip to ankle radiographs in total knee arthroplasty. Clin Orthop Relat Res. https://doi.org/10.1097/00003086-20021100000032(404): 196-202

29. Miller EJ, Pagnano MW, Kaufman KR (2014) Tibiofemoral alignment in posterior stabilized total knee arthroplasty: Static alignment does not predict dynamic tibial plateau loading. J Orthop Res 32(8):1068-1074

30. Moser LB, Hess S, Amsler F, Behrend H, Hirschmann MT (2019) Native non-osteoarthritic knees have a highly variable coronal alignment: a systematic review. Knee Surg Sports Traumatol Arthrosc 27(5):1359-1367

31. Nedopil AJ, Howell SM, Hull ML (2017) What mechanisms are associated with tibial component failure after kinematicallyaligned total knee arthroplasty? Int Orthop 41(8):1561-1569

32. Niki Y, Nagura T, Nagai K, Kobayashi S, Harato K (2018) Kinematically aligned total knee arthroplasty reduces knee adduction moment more than mechanically aligned total knee arthroplasty. Knee Surg Sports Traumatol Arthrosc 26(6):1629-1635

33. Park A, Duncan ST, Nunley RM, Keeney JA, Barrack RL, Nam D (2014) Relationship of the posterior femoral axis of the "kinematically aligned" total knee arthroplasty to the posterior 
condylar, transepicondylar, and anteroposterior femoral axes. Knee 21(6):1120-1123

34. Parratte S, Pagnano MW, Trousdale RT, Berry DJ (2010) Effect of postoperative mechanical axis alignment on the fifteen-year survival of modern, cemented total knee replacements. J Bone Joint Surg Am 92(12):2143-2149

35. Ritter MA, Davis KE, Davis P, Farris A, Malinzak RA, Berend ME, Meding JB (2013) Preoperative malalignment increases risk of failure after total knee arthroplasty. J Bone Joint Surg Am 95(2):126-131

36. Ritter MA, Davis KE, Meding JB, Pierson JL, Berend ME, Malinzak RA (2011) The effect of alignment and BMI on failure of total knee replacement. J Bone Joint Surg Am 93(17):1588-1596

37. Riviere C, Dhaif F, Shah H, Ali A, Auvinet E, Aframian A, Cobb J, Howell S, Harris S (2018) Kinematic alignment of current TKA implants does not restore the native trochlear anatomy. Orthop Traumatol Surg Res 104(7):983-995

38. Riviere C, Iranpour F, Harris S, Auvinet E, Aframian A, Parratte S, Cobb J (2018) Differences in trochlear parameters between native and prosthetic kinematically or mechanically aligned knees. Orthop Traumatol Surg Res 104(2):165-170

39. Rivière C, Lazic S, Boughton O, Wiart Y, Villet L, Cobb J (2018) Current concepts for aligning knee implants: patient-specific or systematic? EFORT Open Rev 3(1):1-6

40. Riviere C, Ollivier M, Girerd D, Argenson JN, Parratte S (2017) Does standing limb alignment after total knee arthroplasty predict dynamic alignment and knee loading during gait? Knee 24(3):627-633

41. Vanlommel L, Vanlommel J, Claes S, Bellemans J (2013) Slight undercorrection following total knee arthroplasty results in superior clinical outcomes in varus knees. Knee Surg Sports Traumatol Arthrosc 21(10):2325-2330

42. Vicente AM, Ballensiefen W, Jönsson JI (2020) How personalised medicine will transform healthcare by 2030: the ICPerMed vision. J Transl Med 18(1):180

43. Victor JM, Bassens D, Bellemans J, Gursu S, Dhollander AA, Verdonk PC (2014) Constitutional varus does not affect joint line orientation in the coronal plane. Clin Orthop Relat Res 472(1):98-104

44. Young SW, Walker ML, Bayan A, Briant-Evans T, Pavlou P, Farrington B (2017) The Chitranjan S Ranawat Award: no difference in 2-year functional outcomes using kinematic versus mechanical alignment in TKA: a randomized controlled clinical trial. Clin Orthop Relat Res 475(1):9-20

Publisher's Note Springer Nature remains neutral with regard to jurisdictional claims in published maps and institutional affiliations. 